\title{
Inotrópicos, não Devem Ser Prescritos?
}

\author{
Antonio Carlos Pereira Barretto, Luiz Guilherme Velloso
}

São Paulo, SP

O inotrópico foi a primeira droga utilizada para tratar quadros de insuficiência cardíaca, tendo Withering administrado a digital há mais de 200 anos, obtendo a compensação do quadro desta síndrome. Nos últimos anos, vários agentes com esta atividade passaram a ser empregados na terapêutica da insuficiência cardíaca congestiva (ICC).

Se não há muitas dúvidas sobre seu valor na terapêutica dos quadros descompensados ${ }^{1-5}$, o mesmo não ocorre quando da sua prescrição de maneira crônica para pacientes compensados ${ }^{6-9}$. A sua prescrição, quando e como, vem sendo objeto de vários artigos e é motivo de intensa controvérsia, o que nos induziu a expor nosso ponto de vista sobre tema tão importante.

No paciente agudamente descompensado e com baixo débito cardíaco há pouca dúvida quanto aos efeitos benéficos do suporte inotrópico. Persistência de quadro congestivo associada a palidez e redução da temperatura cutânea, enchimento capilar extremamente lento, pulsos filiformes, hipotensão arterial, alteração do sensório, anorexia, intolerância alimentar, elevação progressiva dos níveis de uréia e creatinina permitem o diagnóstico do baixo débito, sem a necessidade de monitorização invasiva. Nesta situação de elevada mortalidade e grande sofrimento para o paciente, o uso de suporte inotrópico endovenoso traz melhora imediata e redução acentuada dos sintomas. As catecolaminas dobutamina e dopamina são os medicamentos mais utilizados com esta finalidade.

Em pacientes internados no Hospital Auxiliar de Cotoxó, que recebe do Pronto Socorro do INCOR pacientes descompensados, observamos que a administração de dobutamina em doses de 5 a $10 \mu \mathrm{m} / \mathrm{kg} / \mathrm{min}$ resultou em maior número de altas hospitalares (77\%) que a dopamina (31\%). Taquicardia ventricular sustentada ou piora do quadro hemodinâmico ocorreu em 17\% dos tratados com dopamina, resultando em óbito ou suspensão da administração da droga ${ }^{10}$. É interessante observar que, nesses pacientes com disfunção ventricular crônica, o baixo débito cardíaco pôde ser tratado com inotrópicos sem monitorização especial, em ambiente de enfermaria ${ }^{11}$.

Os inibidores da fosfodiesterase (amrinona, milri-

Instituto do Coração do Hospital das Clínicas - FMUSP

Correspondência: Antonio Carlos Pereira Barretto - Incor - Av. Dr. Enéas C. Aguiar, 44 - 05403-000 - São Paulo, SP

Recebido para publicação em 2/7/97

Aceito em 20/8/97 nona), como a dobutamina, aumentam o débito cardíaco, reduzem a pressão de enchimento ventricular e a resistência vascular sistêmica ${ }^{12}$. No entanto, o custo dos inibidores da fosfodiesterase é bastante superior ao das catecolaminas, o que sugere que em nosso meio possam ser reservadas a casos refratários, para uso em associação com a dobutamina ou em substituição a ela.

Para prevenir a descompensação dos pacientes mais graves, "pulsoterapia" com dobutamina ou amrinona foi utilizada ${ }^{14-16}$. Alguns artigos, entretanto, mostraram que essa conduta era acompanhada de maior mortalidade e deixou de ser preconizada por muitos serviços ${ }^{17}$. Recentemente, um artigo mostrou que seu emprego cuidadoso, pode estabilizar os pacientes, reduzindo as internações em enfermaria ou de emergência, bem como o número de dias internados para compensação. É nosso conceito, que a "pulsoterapia" possa ser usada, entretanto é necessário seguimento muito próximo dos pacientes, pelo mesmo médico, que realmente conhece seu doente ${ }^{16}$. O intervalo para cada prescrição é determinado pelo médico, sendo realizada uma a duas vezes por semana.

A prescrição dos inotrópicos de maneira geral para portadores de ICC melhora sua classe funcional, sua capacidade de exercício e sua qualidade de vida, entretanto a maioria das drogas provoca aumento de mortalidade.

Este aumento da mortalidade é diferente com cada droga e parece ser proporcional à dose. Quanto maior a dose, maior a mortalidade. Com o vesnarinone, resultados preliminares pareciam sugerir que doses menores não induziriam a aumento da mortalidade ${ }^{8}$. Estudo especialmente elaborado para analisar esses aspectos (Estudo VEST), não comprovou este benefício, e mostrou que o aumento da mortalidade era proporcional a dose. Este efeito adverso parece estar relacionado a aumento da estimulação neuro-humoral, aumento da estimulação simpática que induz aumento de arritmias e morte súbita, resultando em menor sobrevida.

Desta forma, não há indicação para a prescrição de inotrópicos para pacientes compensados, sendo exceção os digitálicos, queinduzindoredução da frequiência cardíaca, modulação da estimulação neuro-humoral e redução da estimulação simpática parecem não provocar aumento da mortalidade.

O estudo DIG documentou este aspecto, mostrando que o uso de digoxina não tem impacto sobre a sobrevida ${ }^{18}$. Este resultado pode levar a duas condutas, a não prescrevêla pois não modifica a reduzida sobrevida dos portadores de ICC ou prescrevê-la pelos seus benefícios na melhora da 
função cardíaca, classe funcional, tempo de exercício e redução do número de hospitalizações. O resultado do estudo DIG mostra redução do número de internações (eventos) para aqueles em uso do medicamento. Resultado semelhante já havia sido observado nos estudos RADIANCE e PROVED, que demonstraram que a retirada de digital era acompanhado de piora da ICC, redução no tempo de exercício ou da distância caminhada ${ }^{19,20}$.

A controvérsia sobre o emprego do digital ainda não terminou apesar destes resultados. No estudo DIG, observou-se que o digital aumentava mortalidade daqueles que apresentavam arritmia ventricular. Aguarda-se análise mais detalhada dos subgrupos do estudo DIG para se concluir se todos pacientes podem tomar digital.

Há também dúvida se os digitálicos devam ser prescritos para pacientes com ICC secundária a doença isquêmica, onde alguns artigos mostram que a droga pode aumentar a mortalidade $^{21}$.

Assim, embora não seja medicação que, isoladamente, controle os quadros de insuficiência cardíaca, associado a diuréticos e inibidores da enzima conversora da angiotensina, desempenha papel importante, conforme os resultados mencionados.

Há um ponto mais controverso, que merece discussão. Não devemos utilizar os inotrópicos não digitálicos para tratamento de pacientes compensados?

Consideramos que pelo aumento da mortalidade que induzem, não deveriam ser prescritos para os pacientes estáveis, que compensaram com o tratamento clássico de diuréticos, digital e vasodilatadores. Entretanto, questionamos se não poderia ser prescrito para pacientes com ICC avançada, que não se mantêm compensados, necessitando múltiplas internações, e mesmo para aqueles muito limitados apesar da terapêutica otimizada. Estes pacientes mais graves têm uma qualidade de vida muito ruim, e o emprego dos inotrópicos pode melhorá-la, uma vez que auxiliam a compensação dos pacientes, melhorando o desempenho físico e reduzindo a necessidade de múltiplas internações ${ }^{9,22}$. Assim, nas formas avançadas, talvez o mais importante não seja a quantidade de vida mas a qualidade dela, e isto os inotrópicos melhoram, apesar de poderem reduzir o seu tempo. Esta ação na mortalidade, tem que ser criticamente analisada. No estudo PRIME 2, o ipopamina provocou aumento da mortalidade que passou de $20 \%$ para $24 \%$ em um ano ${ }^{23}$. Este aumento de quatro mortes em um ano, para 100 pacientes, em relação ao grupo controle, significou do ponto de vista estatístico um aumento de mortalidade de $26 \%$. Não que não seja importante este resultado, mas do ponto de vista clínico, para pacientes com qualidade de vida muito ruim, talvez não seja um risco que não se possa correr.

Este tema qualidade versus quantidade de vida vem sendo objeto de discussão em vários países e alguns serviços vêm utilizando os inotrópicos em pacientes com ICC avançada, por considerar que nesta fase da doença não resolve somente prolongar a vida com qualidade tão baixa.

Quanto a pergunta título deste ponto de vista, concluiríamos que há várias indicações para os inotrópicos, nos pacientes descompensados para acelerar sua compensação, reduzindo o desconforto que ela provoca. Para pacientes estáveis, embora sem impacto sobre a mortalidade, o digital estabiliza os pacientes melhorando sua performance e reduzindo os quadros de descompensação. Para pacientes com ICC avançada, os inotrópicos podem ser prescritos para melhorar a qualidade de vida.

\section{Referências}

1. Bendersky R, Chattergee K, Parmley WW et al - Dobutamine in chronic ischemia heart failure: alterations in left ventricular functions and coronary hemodynamic. Am J Cardiol 1981; 48: 554-8.

2. Leier CV, Heban PT, Huss et al - Comparative systemic and regional hemodynamic effects of dopamine and dobutamine in patients with cardiomyopathic heart failure. Circulation 1978; 58: 466-75.

3. Gage J, Rutman H, Lucido D et al - Additive effects of dobutamine and anrinone on myocardial contractility and ventricular performance in patients with severe heart failure. Circulation 1986; 74: 367-73.

4. Goldberg LI - Dopamine: clinical uses of an endogenen catecholamine. NEngl J Med 1974; 291: 707-10.

5. Sanders MR, Kostis JB, Fishman WH - The use of inotropic agents in acute and chronic congestive heart failure. Med Clin North Am 1989; 73: 283-314.

6. Packer M, Carver JR, Rodeheffer et al - Effect of oral miltinone on mortality in severe heart failure. N Engl J Med 1991; 325: 1468-75.

7. Cowoley AJ, Skene AM et al - Treatment of severe heart failure: quantity or quality of life? A trial of enoximone. Br Heart J 1994; 72: 226-30.

8. Feldman AM, Bristow MR, Parmley WW et al - Effects of vesnarinone on morbidity and mortality in patients with heart failure. N Engl J Med 1993; 329: 149-55.

9. Katz SD, Kubo SH, Jessup M et al - A multicenter, randomize a double-blind placebo-controlled trial of pimobendan, a new cardiotonic and vasodilator agent, in patients with severe congestive heart failure. Am Heart J 1992; 123: 95-103.

10. Velloso LGC, Munhoz RT, Bichuete RR et al - Suporte inotrópico em enfermaria geral: comparação entre dobutamina e dopamina. Arq Bras Cardiol 1993; 61(supl II): II-132.

11. Velloso LGC, Munhoz RT, Bichuete RR et al - Dobutamina no baixo débito cardíaco em enfermaria de hospital de retaguarda. Rev Soc Cardiol Est SP 1992; 2(suppl B): 16 .

12. DiBianco R - Acute positive inotropic intervention: the phosphodiesterase inhibitors. Am Heart J 1991; 6(suppl 1): 1871-4.
13. Marcus RH, Raw K, Patel J, Mitha A, Sareli P - Comparison of intravenous amrinone and dobutamine in congestive heart failure due to idiopathic dilated cardiomyopathy. Am J Cardiol 1990; 66: 1107-12.

14. Leier CV, Webel J, Bush CA - The cardiovascular effects of the continuous infusion of dobutamine in patients with severe heart failure. Circulation 1977; 56: 46872 .

15. Unverferth DV, Magorien RD, Lewis RR, Leier CV - Long-term benefit of dobutamine in patients with congestive cardiomyopathy. Am Heart J 1980; 100: 622-30.

16. Mareiro-Nunez AL, Heaney L, Fernandez RN et al - Intermittent inotropic therapy in an outpatient setting: A cost-effective therapeutic modality in patients with refractory heart failure. Am Heart J 1996; 132: 805-8.

17. Salema V, Silva MAD, Hidalgo $P$ et al - Três dias de infusão de dobutamina no tratamento de insuficiência cardíaca congestiva grave. Arq Bras Cardiol 1995; 65(supl I): 87.

18. The Digitalis Investigation Group - The effect of digoxin on mortality and morbidity in patients with heart failure. N Engl J Med 1997; 336: 525-33.

19. Packer M, Gheorghiade M, Young JB et al - Withdrawal of digoxin from patients with chronic heart failure treated with angiotensin-converting-enzyme inhibitors. N Engl J Med 1993; 329: 1-7.

20. Uretsky BF, Young JB, Shahidi E et al - Randomized study assessing the effect of digoxin withdrawal in patients with mild to moderate chronic congestive heart failure. Results of the PROVED trial. J Am Coll Cardiol 1993; 22: 955-62.

21. Benerjee AK, Campbell RWF - Digoxin therapy and survival in heart failure in sinus rhythm. Int J Cardiol 1996; 55: 9-13.

22. Bristow MR, Lewis BD - Low dose inotropic therapy for ambulatory heart failure. Coronary Artery Disease. 1994; 5: 112-8.

23. Hampton Jr, Van Veldhuisen DJ, Kleber FX et al - Randomised study of effect of ibopamine on survival in patients with advanced severe heart failure. Lancet 1997; 349: 971-7. 